\title{
Prognostic Value of Procalcitonin, CRP, Serum Amyloid A, Lactate and IL-6 Markers in Liver Transplant Patients Admitted to ED with Suspected Infection
}

\author{
ALI GÜR ${ }^{1 *}$, HAKAN OGUZTURK ${ }^{2 *}$, ADEM KÖSE $^{3}$, M. GÖKHAN TURTAY ${ }^{*}$, VEYSEL ERSAN $^{4 *}$, \\ YAŞAR BAYINDIR ${ }^{3 *}$, VOLKAN INCE ${ }^{4 *}$, SUKRU GURBUZ ${ }^{2}$ and NESLIHAN YUCEL ${ }^{2}$ \\ ${ }^{1}$ Van Education and Research Hospital, Van, Turkey; \\ ${ }^{2}$ Emergency Medicine Department, Faculty of Medicine, Inonu University, Malatya, Turkey; \\ ${ }^{3}$ Infection Diseases Department, Faculty of Medicine, Inonu University, Malatya, Turkey; \\ ${ }^{4}$ Faculty of Medicine, Liver Transplant Institute, Inonu University, Malatya, Turkey
}

\begin{abstract}
Background/Aim: Infections are one of the most important causes of mortality and morbidity after liver transplantation as in all transplantations. Infectious complications are known to be among the preventable causes with appropriate diagnosis and treatment. So early prediction of the risk of infections will provide an effective approach to determine the local antimicrobial resistance and prevention of specific risk factors. The aim of this study was to deterimne whether specific markers are useful or not to deterimne a suspected infection in patients that have undergone liver transplantation. Patients and Methods: The study included 65 patients with liver transplantation admitted to emergency room with suspicion of infection. These patient's CRP, procalsitonin (PCT), lactate, SAA and $I L-6$ levels were initially measured in the emergency department. The patients were classified to three categories according to culture results; culture-negative, culturepositive and control group. Studying parameters were investigated according to whether the culture was positive or negative in these patients. Results: CRP, PCT, lactate, SAA and IL-6 levels were significanlty high in patients with suspected infeciton when compared to the control group $(p<0.05)$. CRP, PCT and IL-6 levels were higher in the
\end{abstract}

This article is freely accessible online.

*These Authors equally contributed to this study.

Correspondence to: Assoc. Prof. Hakan Oguzturk, Faculty of Medicine, Emergency Medicine Department, Inonu University, Malatya,Turkey.E-mail: oguzturk@hotmail.com

Key Words: Liver transplantation, emergency department, CRP, PCT, lactate, SAA, IL-6. culture-positive group than in the culture-negative group and there was a significant variation $(p<0.05)$. When suspecting an infection evaluating the parameters CRP, PCT and IL-6 was very meaningfull $(p<0.05)$. Conclusion: We can use CRP, PCT, lactate, SAA and IL-6 parameters to identify presence of infection at the liver transplantation patients admitted to the emergency department with suspected infection. If CRP, PCT and IL-6 levels are significantly high we can guess the patient's positive culture.

Infections are the primary cause of mortality and morbidity after liver transplants as with all transplants. Therefore, posttransplant infections are an important problem for centers where this procedure is performed. Bacterial infections are more prevalent when the etiologic distribution of postoperative infections is concerned. These infections are usually observed in the surgical site, the abdomen, the urinary tract, the respiratory tract, and at the site of the catheter (1).

Most prevalent bacterial agents are enteric gram-negative microorganisms such as Escherichia coli, Pseudomonas aeruginosa and Acinetobacter. Among the Gram-positive bacteria, enterococci were the most prevalent. In these infections, parameters such as high fever and leukocytosis would be suppressed due to immunosuppression, so diagnosis is quite difficult and the only available method is to obtain routine culture (2).

Although infectious complications are active causes of morbidity and mortality in these patients, they are considered as preventable factors with accurate diagnosis and treatment. Therefore, early prediction of the infection risk will be an active approach to prevent local antimicrobial resistance and specific risk factors (3). There are changes in the acute protein synthesis of the liver in response to systemic responses to an acute infectious stimulus. There is an 
increase in the synthesis of certain acute phase proteins (AFP), while the synthesis of certain proteins decreases. This acute phase response is the host's response due to infection, tissue damage or trauma and also causes fever with the mediation of certain cytokines (4).

Serious bacterial infection suspicion, without doubt, leads to unnecessary use of antibiotics, prolonged hospital treatment, the increase of resistant bacteria in the society, and the increase in the cost for treatment of simple diseases for the family and the society. Thus, we aimed to investigate whether the markers selected in liver transplant patients who are applied to the emergency department (ED) with early and late period infection suspicion are useful markers.

\section{Materials and Methods}

We prospectively enrolled 65 liver transplant patients who were admitted to the emergency department in Inonu University Turgut Özal Medical Center with the suspicion of infection between 01 January 2015 and 30 August 2015. The study included 65 liver transplant patients who were admitted to the ED with symptoms other than infection as the control group.

Patients under 18 years of age, patients who applied for emergency services within 3 months after the transplant and patients with a trauma history were excluded from the study.

Patients who were admitted to the ED with suspected infection were evaluated with a multidisciplinary approach by the infectious diseases and organ transplantation departments in the hospital. The patients were grouped as culture negative $(\mathrm{n}: 38)$, culture positive $(n: 27)$ and control groups (n:65) after the consultation.

The name, surname, gender, age, the reason for transplant, complaints during application, blood pressure, pulse rate, respiratory rate, presence of immunosuppressive agent, white blood cell count, CPR, Procalcitonin, Lactate, Serum Amyloid A and Interleukin-6 values of the patients who were included in the study were recorded on the patient registration form. The patient diagnosis, treatment and culture results were monitored. Patients in the ER were monitored by the same physician that monitored other patients in the same service. Later, the diagnosis, presence of bacterial reproduction in the culture and duration of hospitalization of these patients were recorded on the patient registration form.

Diagnosis of sepsis was attempted based on sepsis conference criteria.

The obtained data were transferred to the computer media and analyzed with SPSS 17.0 (SPSS, Inc., Chicago, IL) software. Patients were initially divided into two groups: patients with and without infection. These two groups were compared with the Student $\mathrm{T}$ test. Patients were then grouped into culture negative, culture positive and control groups based on culture results. The data was summarized with frequencies (count), percentages $(\%)$, and arithmetic mean \pm standard deviation $(\mathrm{X} \pm \mathrm{SD})$. The normal distribution of the continuous numerical data was determined with "coefficient of variation". Comparison of the parameters of continuous numerical data with normal distribution based on more than two groups was conducted with culture-negative group, culture-positive group and control group ANOVA Tukey Test. Risk analysis was then conducted with Roc Curve. Cut off values were 2.51 for CRP, 0.217 for procalcitonin, 11.75 for lactate, 2.35 for
SAA and 21.14 for IL-6. In all analyzes, the difference or correlation was considered statistically significant when $p<0.05$.

Ethics approval and consent to participate. This retrospective single center case study was approved by the local ethics committee of the Inonu University Hospital Malatya, Turkey, and followed the ethical guidelines of Declaration of Helsinki from 1975.

\section{Results}

A total of $\mathrm{n}: 41$ females $(31.5 \%)$ and $\mathrm{n}: 89$ males $(68.5 \%)$ patients were included in the study; the total number of patients were $130(100 \%)$. When the patients were grouped based on gender; in the culture negative group, there were $\mathrm{n}: 38(39.3 \%)$ individuals; $\mathrm{n}: 15$ were female $(11.5 \%)$ and $\mathrm{n}: 23$ were male $(17.8 \%)$. In the culture positive group, there were n:27 (20.7\%) individuals; n:8 were female $(6.1 \%)$ and $\mathrm{n}: 19$ were male $(14.6 \%)$. In the control group, there were $\mathrm{n}: 65(50 \%)$ individuals; $\mathrm{n}: 18$ were female $(13.9 \%)$ and $\mathrm{n}: 47$ were male $(36.1 \%)$.

The mean age of patients in the study was $44 \pm 13$. The mean age of the culture negative patients was $47 \pm 12.3$, the mean age of the culture positive patients was $43.1 \pm 13.4$, and the mean age of the patients in the control group was $42.3 \pm 13$. No statistically significant difference was found between the mean age of all patient groups and the control group.

The transplant reasons of the patients based on the culturenegative, culture-positive, and the control group are presented in detail in Table I.

The complaints of the patients when they applied to the ED based on the culture negative group, the culture positive group and the control group are detailed in Table II.

Significant differences were found between systolic blood pressure, diastolic blood pressure, pulse rate, respiratory rate, and fever between the infection and control $(p<0.05)$ when the patients were evaluated based on their mean vital signs (Table III).

In contrast, there was no significant difference in the number of white blood cells between the infection group and the control group $(p>0.05)$ (Table III).

When the patients were evaluated based on the mean study parameters, there was a significant difference between CRP, procalcitonin, lactate, serum amyloid A, IL-6 values in the infection group and the control group $(p<0.05)$ (Table III).

Furthermore, there was a significant difference between the infection group and the control group based on the time spent as an inpatient $(p<0.05)$ (Table III).

When the patients were evaluated based on the correlation between the mean CRP values in the groups, a significant difference was found between the values of both procalcitonin and CRP for the culture negative and culture positive groups. The correlations between the parameters and groups are presented in Table IV. 
Table I. The transplant reasons of the groups.

\begin{tabular}{lcccc}
\hline Transplant Reasons & Culture-Negative $(\mathrm{n}=38,29.3 \%)$ & Culture-Positive (n=27, 20.7\%) & Control (n=65, 50\%) & Total (n=130, 100\%) \\
\hline Hepatitis B & $19(14.67 \%)$ & $14(10.13 \%)$ & $35(27.67 \%)$ & $68(52.26 \%)$ \\
Cryptogenic cirrhosis & $1(0.77 \%)$ & $4(3.08 \%)$ & $5(3.85 \%)$ & $10(7.7 \%)$ \\
HBV+HDV & $1(0.77 \%)$ & $3(2.31 \%)$ & $5(3.85 \%)$ & $9(6.93 \%)$ \\
Toxic hepatitis & $3(2.31 \%)$ & $0(0 \%)$ & $4(3.08 \%)$ & $7(5.39 \%)$ \\
Alcoholic cirrhosis & $3(2.31 \%)$ & $0(0 \%)$ & $3(2.31 \%)$ & $6(4.62 \%)$ \\
Primary biliary cirrhosis & $0(0 \%)$ & $2(1.54 \%)$ & $4(3.08 \%)$ & $6(4.62 \%)$ \\
HCC & $4(3.08 \%)$ & $0(0 \%)$ & $2(1.54 \%)$ & $6(4.62 \%)$ \\
Hepatitis D & $3(2.31 \%)$ & $1(0.77 \%)$ & $1(0.77 \%)$ & $5(3.85 \%)$ \\
Hepatitis C & $2(1.54 \%)$ & $1(0.77 \%)$ & $0(1.54 \%)$ & $5(3.85 \%)$ \\
Crohn's disease & $1(0.77 \%)$ & $2(1.54 \%)$ & $3(2.31 \%)$ & $3(2.31 \%)$ \\
Others* & $1(0.77 \%)$ & $1(0.77 \%)$ & & $5(3.85 \%)$ \\
\hline
\end{tabular}

Others*: Portal vein thrombosis, Wilson's disease, Budd-Chiari, lymphoma.

Table II. The application complaints of the groups.

\begin{tabular}{lcccc}
\hline Application complaints & Culture-Negative $(\mathrm{n}=38,29.3 \%)$ & Culture-Positive $(\mathrm{n}=27,20.7 \%)$ & Control $(\mathrm{n}=65,50 \%)$ & Total $(\mathrm{n}=130,100 \%)$ \\
\hline Fever & $31(23.84 \%)$ & $26(20 \%)$ & $0(0 \%)$ & $57(43.84 \%)$ \\
Abdominail pain & $13(10 \%)$ & $4(3.07 \%)$ & $20(15.38 \%)$ & $37(28.45 \%)$ \\
Pain* & $2(1.53 \%)$ & $0(0 \%)$ & $30(23.07 \%)$ & $32(24.61 \%)$ \\
Nausea vomiting & $15(11.53 \%)$ & $9(6.92 \%)$ & $7(5.38 \%)$ & $31(23.84 \%)$ \\
Itching & $5(3.84 \%)$ & $1(0.76 \%)$ & $12(9.23 \%)$ & $18(13.83 \%)$ \\
Weakness & $8(6.15 \%)$ & $6(4.61 \%)$ & $0(0 \%)$ & $14(10.76 \%)$ \\
Cough-sputum & $6(4.61 \%)$ & $8(6.15 \%)$ & $0(0 \%)$ & $14(10.76 \%)$ \\
Dysuria & $9(6.92 \%)$ & $3(2.30 \%)$ & $0(0 \%)$ & $12(9.23 \%)$ \\
Dyspnoea & $6(4.61 \%)$ & $3(2.30 \%)$ & $0(0 \%)$ & $9(6.92 \%)$ \\
Diarrhea & $3(2.30 \%)$ & $2(1.53 \%)$ & $4(3.07 \%)$ & $9(6.92 \%)$ \\
Anorexia & $0(0 \%)$ & $0(0 \%)$ & $5(3.84 \%)$ & $5(3.84 \%)$ \\
Others** & $0(0 \%)$ & $0(0 \%)$ & $7(5.38 \%)$ & $7(5.38 \%)$ \\
\hline
\end{tabular}

*Pain: Headache, Side pain, Body pain, Chest pain, Arm and Leg pain, Joint pain. **Others: Any symptom other than infection symptoms.

When the patients were evaluated based on the diagnosis, it was observed that the patients in the culture negative group were most frequently diagnosed with pneumonia while the patients in the culture negative group were most frequently diagnosed with cholangitis (Table V).

In the culture-positive group, $E$. coli, one of the microorganisms that reproduce in culture, was found in 14 $(51.85 \%)$ patients (Table VI).

When the patients were evaluated based on the mortality rates, it was observed that a total of 8 patients $(6.0 \%)$ deceased despite all treatments (Table VII).

When the increase in the infection risk was evaluated based on the cut-off values of the parameters in culturenegative and culture-positive groups, it was found that the infection risk increased 3.75 (1.08-13.02) times when the cut-off value of CRP was determined as 2.51 and the parameter was considered as significant. When the cut-off value of procalcitonin was 0.217 , there risk of infection was expected to be $11.0(95 \% \mathrm{CI}=2.81-42.94)$ times higher and the parameter was considered as significant $(p<0.05)$. When the cutoff value of lactate was 11.75 , a $0.84(95 \% \mathrm{CI}=0.30$ 2.33) fold increase in risk of infection was expected and the parameter was not statistically significant $(p>0.05)$. When the cut-off value of SAA was 2.35, a 2.56 (95\% CI=0.79-8.3) fold increase in infection risk was expected and the parameter was not significant $(p>0.05)$. When the cut-off value of IL-6 was 21.145 , a $3.75(95 \% \mathrm{CI}=1.08-13.02)$ fold increase in the risk of infection was expected and the parameter was considered significant $(p>0.05)$ (Table VIII).

Sensitivity and Specificity of CRP and SAA were determined when the parameters in culture negative and culture positive groups were examined based on the 
Table III. Mean and standard deviation for the infection group and the control group.

\begin{tabular}{lccc}
\hline Parameters & $\begin{array}{c}\text { Infection } \\
\text { Group } \\
\text { (Mean } \pm \text { SD) }\end{array}$ & $\begin{array}{c}\text { Control } \\
\text { Group } \\
\text { (ortalama } \\
\pm \text { SD) }\end{array}$ & $p$-Value \\
& & & \\
& & & \\
Vital Signs & $121 \pm 22$ & $128 \pm 14$ & 0.042 \\
$\quad$ Systolic blood pressure (mmHg) & $76 \pm 11$ & $82 \pm 7$ & 0.001 \\
Diastolic blood pressure (mmHg) & $88 \pm 23$ & $78 \pm 9$ & 0.001 \\
Pulse rate (atim/dakika) & $20 \pm 3$ & $19 \pm 2$ & 0.016 \\
Respiratory rate (/dakika) & $36.8 \pm 0.9$ & $35.9 \pm 0.2$ & 0.001 \\
Fever ( ${ }^{\circ}$ C) & & & \\
Laboratory Results & $8 \pm 5$ & $7 \pm 2$ & 0.108 \\
$\quad$ White Cells (10 $3 / \mathrm{M})$ & & & \\
Parameters & $5.7 \pm 5.2$ & $0.5 \pm 1$ & 0.001 \\
$\quad$ CRP (mg/dL) & $10.1 \pm 20.2$ & $2 \pm 2.7$ & 0.002 \\
Procalcitonin (ng/ml) & $18 \pm 16$ & $13 \pm 5$ & 0.009 \\
Lactate (mg/dL) & $7 \pm 7.8$ & $0.5 \pm 1.1$ & 0.001 \\
Serum amyloid A (mg/L) & $518 \pm 163$ & $18 \pm 36$ & 0.003 \\
IL 6 (pg/mL) & $16 \pm 17$ & $2 \pm 4$ & 0.001 \\
Duration of hospitalization (day) & & & \\
\hline & &
\end{tabular}

$p$ : Comparison of infection group with control group.

Sensitivity, Specificity, Positive Predictive Value (PPD) and Negative Predictive Value (NPD) (Table IX).

\section{Discussion}

Approximately 40,000 organ transplants are performed per year globally and the annual survival rate is $85-90 \%$ and the 5 -year survival rate is $70-75 \%$. Among these transplants, liver transplants are the second most common after kidney transplants (5). The frequency of systemic infections after liver transplants is prominent and the lifelong use of immunosuppressive drugs is necessary for post-transplant patients. These infections are associated with increased mortality and morbidity. Thus, it is necessary to diagnose the infections induced by microorganisms early in liver transplant patients and to determine the treatment approach as soon as possible (6).

Novel laboratory tests are needed to diagnose serious infections, detect the presence and type of pathogens early, and treat these infections in transplant patients (7). In the present study, we investigated the diagnostic value of CRP, Procalcitonin, Lactate, Serum Amyloid A and IL-6 parameters for the risk of infection in order to perform an early infection diagnosis and immediately start treatment in liver transplant patients.

In a study conducted by Chen et al., microorganisms were reproduced at the catheter site in 25 of the 55 liver transplant patients with the suspicion of infection in catheter site. 36 patients $(65.4 \%)$ were male, $19(34.6 \%)$ were female and the mean age was $53(8)$. In another study, it was found that 135 (93\%) liver transplant patients had clinically significant infections. $81(60 \%)$ of these patients were male and the mean age was 52 (range $=18-69)(9)$.

In the present study, 89 patients $(68.5 \%)$ were male, 41 (31.5) were female and the mean age was $44 \pm 13$. Although the gender distribution of our patients was similar to the studies found in the literature, the mean age of our patient population was younger than that found in the literature.

In the USA, among liver transplant indications, 30\% had $\mathrm{HCV}, 15 \%$ were alcohol users, $10 \%$ had primary biliary cirrhosis, $9 \%$ had cryptogenic cirrhosis, $5 \%$ had autoimmune hepatitis, $4 \%$ had other viral hepatitis, malignancy was observed in $1 \%$ and other causes were observed in $13 \%$. Similar data were observed in Europe. In Turkey, more than $60-70 \%$ of transplant recipients had chronic viral hepatitis B (HBV)-related cirrhosis (10). When we considered the transplant reasons among our patients, it was observed that liver transplant patients received the transplant mostly due to $\mathrm{HBV}$, followed by cryptogenic cirrhosis. This ratio was similar to the national literature and certain other studies.

In a previous study, $93.3 \%$ of the patients who were diagnosed with an infection applied to emergency services with a complaint (11). In the present study, 57 (88\%) patients had complaint of fever, $24(37 \%)$ had nausea and vomiting, and $17(26 \%)$ patients had abdominal pain. The fact that the complaint of fever was the most common complaint in our study was consistent with other findings in the literature.

In the present study, pneumonia was detected as the most common infection in liver transplant patients with infection and this finding was consistent with the literature (12). In our study, $93 \%$ of the reproduced agents were bacteria, which was similar to the findings in the literature.

$E$. coli was the most common gram-negative bacterium reproduced in post-transplant blood culture, followed by $K$. pneumoniae and $P$. aeruginosa. This rate varies between $13 \%$ and $44 \%$ in some centers (13). In the present study, bacterial agents reproduced in blood cultures of 27 of 65 (41.4\%) liver transplant patients who were tested for a suspected infection. This rate was consistent with the literature. When the reproduced factors were examined, it was observed that $E$. coli reproduced in 14 patients $(51.8 \%)$, Staphylococcus aureus reproduced in $22.2 \%$ and Klebsiella pneumoniae reproduced in $11.1 \%$ of the patients. The other factors reproduced in our study were similar to those observed in liver transplant patients in other studies in the literature.

Clinical manifestations of fever/hypothermia, unexplained tachycardia or tachypnea, peripheral vasodilatation symptoms, unexplained shock, impaired mental status should be a reason to consider infection. Leukocytosis or leukopenia, unexplained lactic acidosis, unexplained changes in renal or hepatic 
Table IV. Parameter mean and standard deviations between groups.

\begin{tabular}{|c|c|c|c|c|c|c|}
\hline Parameters & Culture-Negative $($ Mean \pm SD) & Culture-Positive $($ Mean \pm SD) & Control (Mean \pm SD) & $p$-Value $^{1}$ & $p$-Value ${ }^{2}$ & $p$-Value ${ }^{3}$ \\
\hline CRP & $4.6 \pm 4.7$ & $7.2 \pm 5.5$ & $0.5 \pm 1$ & 0.015 & 0.001 & 0.001 \\
\hline Procalsitonin & $2.6 \pm 3.0$ & $20.5 \pm 28.3$ & $2 \pm 2.7$ & 0.001 & 0.969 & 0.001 \\
\hline Lactate & $15 \pm 9$ & $22 \pm 22$ & $13 \pm 5$ & 0.053 & 0.517 & 0.001 \\
\hline Serum amyloid A & $6.8 \pm 9$ & $7.2 \pm 6$ & $0.5 \pm 1.1$ & 0.953 & 0.001 & 0.001 \\
\hline IL 6 & $103 \pm 250$ & $1104 \pm 1888$ & $18 \pm 36$ & 0.001 & 0.880 & 0.001 \\
\hline
\end{tabular}

${ }^{1}$ Comparison of culture negative group with culture positive group; ${ }^{2}$ Comparison of culture negative group with control group; ${ }^{3}$ Comparison of culture positive group with control group.

Table V. The diagnosis of culture negative group and culture positive group.

\begin{tabular}{lllc}
\hline Diagnosis & $\begin{array}{c}\text { Culture } \\
\text { Negative } \\
(\mathrm{n}=38)\end{array}$ & $\begin{array}{c}\text { Culture } \\
\text { Positive } \\
(\mathrm{n}=27)\end{array}$ & $\begin{array}{c}\text { Total } \\
(\mathrm{n}=65)\end{array}$ \\
\hline Pneumonia & $6(9.23 \%)$ & $10(15.38 \%)$ & $16(24.61 \%)$ \\
Sepsis & $1(1.53 \%)$ & $11(16.92 \%)$ & $12(18.45 \%)$ \\
Cholangitis & $9(13.84 \%)$ & $0(0 \%)$ & $9(13.84 \%)$ \\
Urinary tract infection & $6(9.23 \%)$ & $2(3.07 \%)$ & $8(12.3 \%)$ \\
Bilioma & $5(7.69 \%)$ & $2(3.07 \%)$ & $7(10.76 \%)$ \\
Intrahepatic abse & $4(6.16 \%)$ & $1(1.53 \%)$ & $5(7.69 \%)$ \\
Others* & $7(10.76 \%)$ & $1(1.53 \%)$ & $8(12.3 \%)$ \\
\hline
\end{tabular}

*Others: Catheter infection, Cellulitis, Pancreatitis, Cholecystitis.

Table VI. Cultured microorganisms in culture.

\begin{tabular}{lrr}
\hline Microorganisms & $\mathrm{n}: 27$ & $(100 \%)$ \\
\hline Stafilokokus Aureus & 6 & $22.22 \%$ \\
Acinetobakter & 1 & $3.70 \%$ \\
Klebsiella & 3 & $11.11 \%$ \\
E. Coli & 14 & $51.85 \%$ \\
Candida & 1 & $3.70 \%$ \\
Aeromanas Hidrofilia & 1 & $3.70 \%$ \\
Psödomonas Aeroginoza & 1 & $3.70 \%$ \\
\hline
\end{tabular}

function, thrombocytopenia or disseminated intravascular coagulation (DIC), elevated oxygen consumption, low systemic vascular resistance/increased cardiac output should also be considered as indications of infection (14).

In the present study, there was a significant difference between the infection group and the control group patients based on systolic blood pressure, diastolic blood pressure, pulse rate, respiration rate and fever. These parameters were
Table VII. Mortality rates of patients.

\begin{tabular}{|c|c|c|c|c|}
\hline Mortality & $\begin{array}{c}\text { Culture - } \\
\text { Negative } \\
(\mathrm{n}: 38,29.3 \%)\end{array}$ & $\begin{array}{c}\text { Culture - } \\
\text { Positive } \\
\text { (n:27, 20.7\%) }\end{array}$ & $\begin{array}{c}\text { Control } \\
(\mathrm{n}: 65,50 \%)\end{array}$ & $\begin{array}{c}\text { Total } \\
\text { (n:130,100\%) }\end{array}$ \\
\hline Live & $36(27.8 \%)$ & $21(16.2 \%)$ & $65(50 \%)$ & $122(94 \%)$ \\
\hline Dead & $2(1.5 \%)$ & $6(4.5 \%)$ & $0(0 \%)$ & $8(6.0 \%)$ \\
\hline $\begin{array}{l}\text { Table VIII } \\
\text { increase. }\end{array}$ & I. Cut-off value & of parameters $i$ & in infectious pat & atients and risk \\
\hline Parameters & Cut-off & f value & $\begin{array}{c}\text { Odss Ratio- } \\
95 \% \mathrm{CI}\end{array}$ & $p$-Value \\
\hline CRP & 2. & .51 & $3.75(1.08-13.02)$ & 0.031 \\
\hline Prokalsitor & $\operatorname{nin}$ & 217 & $.0(2.81-42.94)$ & 0.001 \\
\hline Laktat & 11. & & $.84(0.30-2.33)$ & 0.750 \\
\hline SAA & 2. & 35 & $.56(0.79-8.3)$ & 0.109 \\
\hline IL-6 & 21. & & $.75(1.08-13.02)$ & 0.031 \\
\hline
\end{tabular}

significant in distinguishing infected and non-infected liver transplant patients.

The CRP value was significantly $(p<0.001)$ in infected patients with liver transplant as in our study (9). This enforced the idea that CRP was a parameter that could be used as an indication of infection in liver transplant patients.

Procalcitonin (PCT) is a protein that responds to bacterial infections and is used as a sepsis marker in diagnosis and treatment infections in post-transplant cases (15). Sudhir et al. emphasized that PCT was an excellent indicator of sepsis and that the addition of the PCT test to standard techniques in critical care of the patients with sepsis would increase the accuracy of the diagnosis (16). Studies on SAA, PCT and IL6 also compared the SAA and PCT values between infected 
Table IX. Parameters' Sensitivity, specificity, PPD and NPD.

\begin{tabular}{|c|c|c|c|c|}
\hline Parameters & Sensitivity & Specificity & PPD (95CI\%) & NPD (95CI\%) \\
\hline CRP & $71 \%$ & $93.8 \%$ & $38.4 \%(0.30-0.47)$ & $61.5 \%(0.52-0.69)$ \\
\hline Procalsitonin & $66.2 \%$ & $90.8 \%$ & $37.6 \%(0.29-0.46)$ & $62.3 \%(0.53-0.70)$ \\
\hline Lactate & $60 \%$ & $47.1 \%$ & $56.9 \%(0.47-0.68)$ & $43.0 \%(0.34-0.52)$ \\
\hline SAA & $71 \%$ & $93.4 \%$ & $37.6 \%(0.29-0.46)$ & $62.3 \%(0.53-0.70)$ \\
\hline IL-6 & $70.8 \%$ & $76.9 \%$ & $46.9 \%(0.38-0.55)$ & $53.0 \%(0.44-0.61)$ \\
\hline
\end{tabular}

and non-infected patients and found that SAA, IL-6 and PCT values were significant in indicating infection (17). Similar results were obtained in the present study.

Although certain studies suggested that lactate is insignificant in indicating infections, in the present study, it was found that the lactate value was significantly associated with infection, when the lactate values of infected livertransplant patients were compared with those of the control group patients (18). Thus, the present study differed from other studies and established that the lactate value was a significant parameter in the indication of infections.

The CRP cut-off value was determined as 10.4, CRP sensitivity was determined as $36 \%$ and specificity was determined as $96.6 \%$ with $95 \% \mathrm{CI}=1.4-16.8$ in 30 liver transplant patients with sepsis. In the present study, when the cut-off value of CRP was determined as 2.51 , the sensitivity was $71 \%$ and specificity was $93.8 \%$ with $95 \% \mathrm{CI}=1.08$ 13.02. The CRP PPD was determined as $38.4 \%(0.30-0.47)$ and NPD was determined as $61.5 \%(95 \% \mathrm{CI}=0.52-0.69)$. Thus, it could be estimated that when the CRP value is above 2.51 , the risk of infection increased 3.75 times and if the CRP value is below the abovementioned value, the possibility of no infection would be $61.5 \%$.

In another study, the cut-off value of procalcitonin was determined as 9.3 and the sensitivity was determined as $32 \%$ and the specificity as $96.6 \%$ with $95 \% \mathrm{CI}=1.2-69.2$ (19). Furthermore, in another study, sensitivity was determined as $70 \%$, specificity was determined as $91 \%$, PPV as $90 \%$ and NPV as $72 \%$ (30) for sepsis patients when the upper limit for PCT was determined as $1.00 \mathrm{ng} / \mathrm{ml}$. In a meta-analysis, 6 studies and 77 liver transplant patients were scrutinized. Meta-analysis demonstrated that the procalcitonin cut-off value was $6.12(3.79-9.88)$. The sensitivity and specificity of procalcitonin were $90 \%$ and $85 \%$, respectively (21).

In the present study, when PCT cut-off value was determined as 0.217 , the sensitivity was $66.2 \%$ and specificity was $90.8 \%$ with $95 \% \mathrm{CI}=2.81-42.94$. PCT PPD was $37.6 \%(95 \% \mathrm{CI}=0.29-0.46)$ and $\mathrm{NPD}$ was $62.3 \%$ (95\% CI=0.53-0.70). When the PCT value was above 0.217 , a 11-fold increase in the risk of infection was estimated and the possibility of no infection was estimated as $62.3 \%$ when the PCT was below this value.
In a previous study, it was found that ROC curve value of the lactate level in liver transplant patients with infection was $0.55(95 \% \mathrm{CI}=0.40-0.70)$. In the same study, the effect of lactate level on mortality was determined and it was found that the effect on mortality was insignificant $(p>0.05)(22)$.

In our study, however, with a lactate cut-off value of 11.75 , it was determined that lactate (0.84) was not significant in the indication of infection risk $(95 \% \mathrm{CI}=0.30$ 2.33 ). However, when the lactate value was 11.75 , the sensitivity was $60 \%$ and the specificity was $47.1 \%$. Thus, it could be argued that the increase in the infection risk could not be determined based on the lactate value.

In another study, patients were divided into 2 groups to determine the risk of infection and their SAA levels were examined. It was concluded that the SAA value was an insignificant marker for the severity of infection. (23)

In our study, it was determined that SAA was 2.56 (0.798.3 ) with a cut-off value of 2.35 , and it was not significant in determining the risk of infection. However, sensitivity was $71 \%$ and specificity was $93.4 \%$ when the SAA value was 2.35. SAA PPD was $37.6 \%(0.29-0.46)$ and NPD was $62.3 \%$ (0.53-0.70). Thus, it could be estimated that the noninfection rate was $62.3 \%$ when SAA values were below 2.35 .

In a previous study, when IL-6 cut-off value was 3.05 , it was found that sensitivity was $96 \%$ and specificity was $36 \%$ (24). In the present study, when IL-6 cut-off value was 21.14, sensitivity was $70.8 \%$ and specificity was $76.9 \%$ with 95\% CI $=1.08-13.02$. IL-6 PPD was 46.9\% (0.38-0.55) and NPD was $53.0(0.44-0.61)$. Thus, it could be estimated that when the IL-6 value was above 21.14 , infection risk increased 3.75 times.

The patient mortality rates of $12-16 \%$ (25) found in the literature were similar to the $12 \%$ mortality rate determined in our study.

\section{Conclusion}

In conclusion, CRP, PCT, Lactate, SAA and IL-6 parameters can be used to determine the presence of infection in liver transplant patients who applied to ER with suspicion of infection. Culture reproduction in patients could be predicted when patient CRP, PCT and IL-6 levels significantly increase. 


\section{Ethics Approval and Consent to Participate}

This retrospective single center case study was approved by the local ethics committee of the Inonu University Hospital Malatya, Turkey, and followed the ethical guidelines of Declaration of Helsinki from 1975.

Availability of Data and Material All presented data are available and can be requested from the Authors.

\section{Conflicts of Interest}

The Authors declare that they have no competing interests.

\section{References}

1 Moreno A, Cervera C, Gavaldá J, Rovira M, Cámara R, Jarque I, Montejo M, Torre-Cisneros J, Miguel Cisneros J, Fortún J, López-Medrano F, Gurguí M, Muñoz P, Ramos A and Carratalá $\mathrm{J}$ : Bloodstream infections among transplant recipients: results of a nationwide surveillance in Spain. Am J Transplant 7: 25792586, 2007.

2 Van DC and Blumberg EA: Multidrug resistant gramnegative bacteria in solid organ transplant recipients. Am J Transplant 9(Suppl 4): 27-34, 2009.

3 Watt KD, Pedersen RA, Kremers WK, Heimbach JK and Charlton MR: Evolution of causes and risk factors for mortality postliver transplant: results of the NIDDK long-term follow-up study. Am J Transplant 10: 1420-1427, 2010.

4 Gabay C and Kushner I: Acute-phase proteins and other systemic responses to inflamation. N Engl J Med 340: 448-454, 1999.

5 Fishman JA: Infection in solid-organ transplant recipients. N Engl J Med 357: 2601e14, 2007.

6 Chong AS and Alegre ML: The impact of infection and tissue damage in solid-organ transplantation. Nat Rev Immunol 12: 459e71, 2012

7 Becker KL, Nylen ES, White JC, Muller B and Snider RH Jr: Clinical review 167: Procalcitonin and the calcitonin gene family of peptides in inflammation, infection, and sepsis: a journey from calcitonin back to its precursors. J Clin Endocrinol Metab 89: 1512e25, 2004.

8 Chen J, Wang Y, Shen Z, Zhu Z, Song Y and Han R: Early diagnostic value of plasma PCT and BG assay for CRBSI after OLT. Transplant Proc 43(5): 1777-1779, 2011.

9 van den Broek MA, Olde Damink SW, Winkens B, Broelsch CE, Malagó M, Paul A and Saner FH: Procalcitonin as a prognostic marker for infectious complications in liver transplant. Recipients in an intensive care unit. Liver Transpl 16: 402-410, 2010.

10 Okuda K: Hepatocelluler carcinoma. J Hepatol 32: 225-237, 2000 .

11 Cooper D, Sharples L, Cornelissen J, Wallwork J, Alexander G and Trull A: Comparison between procalcitonin, serum amyloid $\mathrm{A}$, and C-reactive protein as markers of serious bacterial and fungal infections after solid organ transplantation. Transplant Proc 33: 1808e10, 2001.

12 Sudhi U, Venkatachalaiah RK, Kumar TA, Rao MY and Kempegowda P: Significance of serum procalcitonin in sepsis Indian. J Crit Care Med 15(1): 1-5, 2011.

13 Al-Hasan MN, Razonable RR, Eckel-Passow JE and Baddour LM: Incidence rate and outcome of Gram-negative bloodstream infection in solid organ transplant recipients. Am J Transplant 9: 835-843, 2009.

14 Singer M, Deutschman CS, Seymour CW, Shankar-Hari M, Annane D, Bauer M, Bellomo R, Bernard GR, Chiche JD, Coopersmith CM, Hotchkiss RS, Levy MM, Marshall JC, Martin GS, Opal SM, Rubenfeld GD, van der Poll T, Vincent JL and Angus DC: The Third International Consensus Definitions for Sepsis and Septic Shock (Sepsis-3). JAMA 315(8): 801-810, 2016.

15 Meisner M: Procalcitonin-a new, innovative infection parameter biochemical and clinical aspects. Third edition. Stuttgart, New York, Thieme, 2000.

16 De Villa VH, Lo CM and Chen $\mathrm{CH}$ : Ethics and rationale of living-donor liver transplantation in Asia. Transplantation 15(Suppl): 2-5, 2003.

17 Coelho MC, Tannuri U, Tannuri AC, Reingenheim C and Troster EJ: Is procalcitonin useful to differentiate rejection from bacterial infection in the early post-operative period of liver transplantation in children? Pediatr Transplant 13: 1004-1006, 2009.

18 Castelli GP, Pognani C, Meisner M, Stuani A, Bellomi D and Sgarbi L: Procalcitonin and C-reactive protein during systemic inflammatory response syndrome, sepsis and organ dysfunction. Critical Care 8: 234-242, 2004.

19 Paugam-Burtz C, Albuquerque M, Baron G, Bert F, Voitot H, Delefosse D, Dondero F, Sommacale D, Francoz C, Hanna N, Belghiti J, Ravaud P, Bedossa P,Mantz J and Paradis V: Plasma proteome to look for diagnostic biomarkers of early bacterial sepsis after liver transplantation: a preliminary study. Anesthesiology 112(4): 926-935, 2010.

20 Tsangaris I, Plachouras D, Kavatha D, Gourgoulis G.M, Tsantes A, Kopterides P, Tsaknis G, Dimopoulou I, Orfanos S, GiamarellosBourboulis E, Giamarellou H and Armaganidis A: Diagnostic and prognostic value of procalcitonin among febrile critically ill patients with prolonged ICU stay. BMC Infect Dis 9: 213, 2009.

21 Yu XY, Wang Y, Zhong H, Dou QL, Song YL and Wen H : Diagnostic Value of Serum Procalcitonin in Solid Organ Transplant Recipients: A Systematic Review and Meta-analysis. Transplant Proc 46(1): 26-32, 2014.

22 Cardoso N, Silva T, Cagnolati D, Freitas T, Mente ED, BasileFilho A and Castro e Silva O: Can joint analysis of postoperative MELD, base excess and blood lactate levels be used as an index of postoperative outcome for patients submitted to liver transplantation? Acta Cir Bras 28: 54-60, 2013.

23 Çelebi S, Bulur N, Hacımustafaoğlu M, Özakın C, Çakır D, Bozdemir ŞE and Çetin BŞ: Comparison of C-Reactive Protein, Procalcitonin and Serum Amyloid-A Levels in Children with Bacterial Infections. J Pediatr Inf 7: 147-156, 2013.

24 Grammatikopoulos T, Dhawan A, Bansal S, Wade J, Sherwood R, Dew T, Heaton N and Verma A: Baseline evaluation of serum markers of inflammation and their utility in clinical practice inpaediatric liver transplant recipients. Clin Res Hepatol Gastroenterol 36: 365-370, 2012.

25 Perrakis A, Yedibela S, Schellerer V, Hohenberger W and Müller $\mathrm{V}$ : Procalcitonin in the setting of complicated postoperative course after liver transplantation. Transplant Proc 42(10): 41874190, 2010 . 